\title{
Cardiovascular outcomes with angiotensin II receptor blockers: clinical implications of recent trials
}

This article was published in the following Dove Press journal:

Vascular Health and Risk Management

23 June 2011

Number of times this article has been viewed

\section{Magnus Baumhäkel \\ Michael Böhm}

Department of Cardiology, University Hospital of Saarland, Homburg Saar, Germany
Correspondence: Magnus Baumhäkel Department of Cardiology, University Hospital of Saarland, Kirrbergerstrasse, Homburg Saar 6642I, Germany

$\mathrm{Tel}+490684$ II 6 I 32I 2

Fax +490 684 II6। 32।4

Email magnus@baumhaekel.de
Abstract: Activation of the renin-angiotensin system plays a major role in cardiovascular morbidity and mortality. Recently, angiotensin II receptor blockers (ARBs) have been the subject of a number of large clinical cardiovascular outcome trials, indicating beneficial effects of ARBs with more than 384,000 patient-years of data in different cardiovascular diseases along the cardiovascular continuum, from patients with risk factors, through high cardiovascular risk, to patients with heart failure. This article reviews the implications of these trials for the optimal management of cardiovascular risk.

Keywords: angiotensin receptor blockers, clinical trials, cardiovascular outcome

\section{Introduction}

Cardiovascular diseases remain highly prevalent in western industrialized countries and are estimated to cause more than 4.3 million cardiovascular deaths per year in Europe. ${ }^{1}$ While lifestyle intervention is essential for risk reduction, pharmacological intervention is further necessary to improve individual cardiovascular risk factors, such as lipid status, diabetes, and hypertension. Among the commonly used antihypertensives (diuretics, $\beta$-blockers, calcium channel blockers, or angiotensin-converting enzyme inhibitors), in recent years the angiotensin II receptor blockers (ARBs) have been the focus of a number of large clinical outcome trials in patients at various stages of the cardiovascular continuum. This article reviews the implications of these trial results for the optimal management of cardiovascular risk.

\section{Continuum of cardiovascular disease progression}

The concept of a cardiovascular disease continuum was first described in the early 1990s, and has since become established as a convenient tool to understand the progressive nature of cardiovascular pathophysiology. ${ }^{2-5}$ Under this paradigm, cardiovascular events are the late complications in a sequence of steps of a progressive pathogenic process, beginning with endothelial dysfunction and manifest atherosclerotic lesions, which progress to target organ damage and end-organ failure.

The concept of the cardiovascular disease continuum has implications for assessing the clinical significance of trial results, because the patients recruited will typically be placed at a specific stage on the continuum, ie, those with cardiovascular risk factors, those with or without manifest end-organ damage, or those already having a history of cardiovascular events. Furthermore, the sizes of the populations with particular manifestations of cardiovascular risk factors or even diseases decrease along the 
continuum (Figure 1). Pathophysiological mechanisms alter along the cardiovascular continuum, but activation of the renin-angiotensin system with increased angiotensin II concentrations has been implicated at every stage and is therefore a prime candidate for intervention.

Angiotensin II contributes to many of the pathogenic processes that initiate and perpetuate cardiovascular disease. Key effects are the synthesis of reactive oxygen species and increased oxidative stress leading to endothelial dysfunction as the initial step in atherosclerosis. ${ }^{6}$ Additional pathogenic actions of angiotensin II include: vascular cell proliferation and migration of smooth muscle cells; expression of adhesion molecules and chemokines that mediate adhesion and migration of the monocytes in the vascular wall; decrease in fibrinolysis; increase in platelet aggregation; and increase in procoagulative state through activation of plasminogen activator inhibitor 1 expression. ${ }^{6,7}$ Given the pivotal role played by the renin-angiotensin system in cardiovascular disease, treatment with angiotensin-converting enzyme inhibitors or ARBs provides protection throughout the cardiovascular continuum.

\section{Outcome studies with ARBs}

Clinical trials have evaluated the effects of ARBs in primary and secondary prevention. Although clinical trials have been conducted in patients with myocardial infarction, the study population could be further divided into patients at the early stages of the cardiovascular continuum, patients at high risk of cardiovascular disease, and patients with left ventricular dysfunction or even chronic heart failure.

\section{Early stages of the cardiovascular continuum}

Three ARB outcome studies recruited patients at a relatively early stage of the cardiovascular continuum with hypertension and additional risk factors. In the Losartan Intervention For Endpoint (LIFE) reduction in hypertension study, over 9000 patients with moderate to severe hypertension (baseline blood pressure 174/98 $\mathrm{mmHg}$ ) and left ventricular hypertrophy were included. ${ }^{8}$ Compared with treatment using atenolol, the ARB losartan improved the primary composite endpoint (cardiovascular mortality, stroke, and myocardial infarction) significantly (relative risk [RR]: 0.87, 95\% confidence interval [CI]: $0.77-0.98, P=0.021$ ) with similar reductions in blood pressure. However, due to side effects, $\beta$-blockers have recently been debated as a first-line treatment in patients with hypertension and are no longer recommended in some countries. ${ }^{9}$ Thus, the results of the LIFE trial may be somewhat limited for clinical practice regarding atenolol as the comparator substance.

The Valsartan Antihypertensive Long-term Use Evaluation (VALUE) trial randomized 15,313 patients with

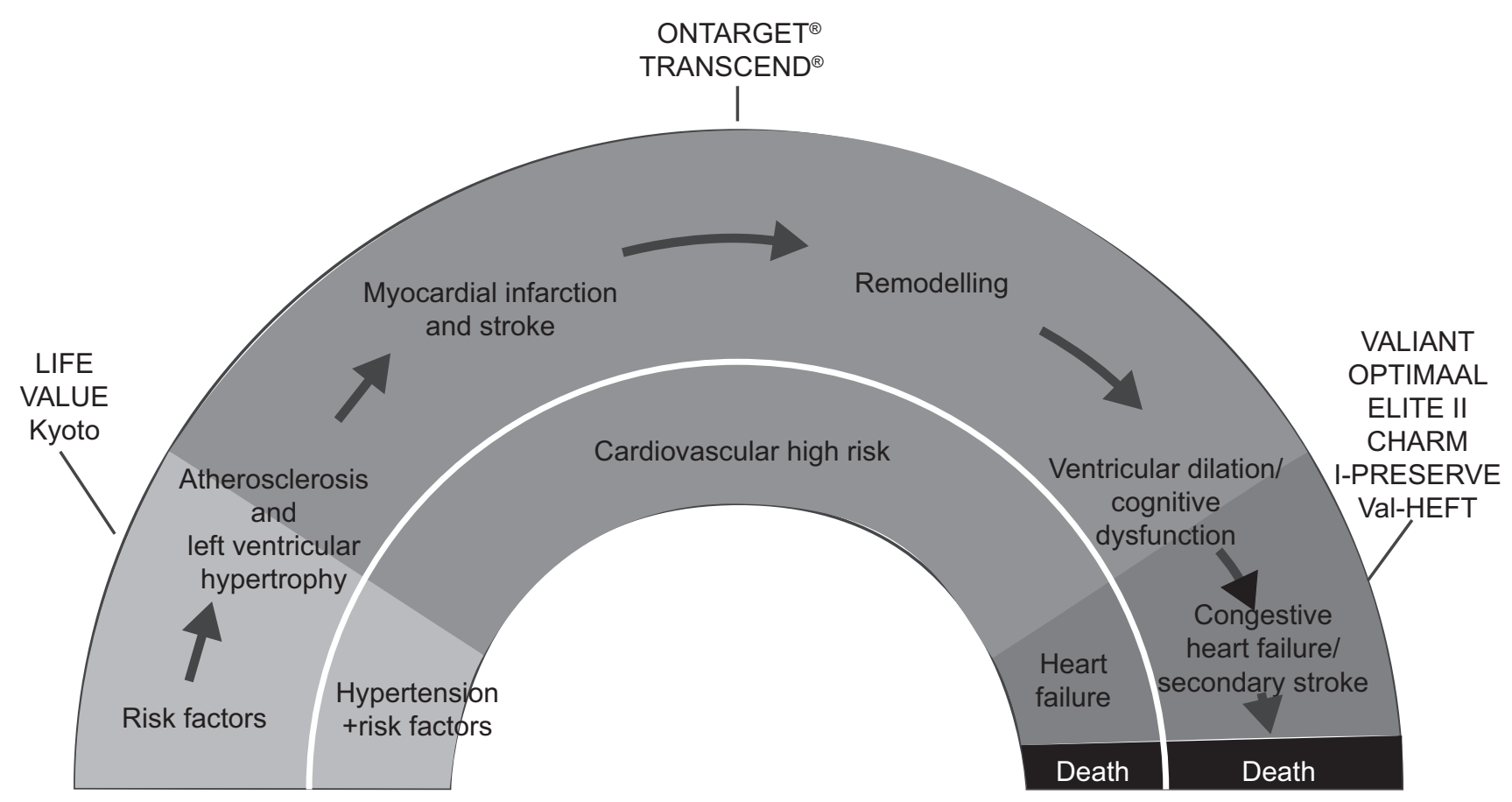

Figure I Angiotensin receptor blocker clinical trials along the cardiovascular continuum. Abbreviations: HF, heart failure; RF, risk factor(s). 
hypertension and additional risk factors (45.8\% coronary heart disease, $31.7 \%$ diabetes, $33.0 \%$ high cholesterol) to either valsartan or amlodipine. Although the two regimens were similar on the primary composite outcome, there was a difference in favor of amlodipine in the early stages of the trial that may be explained by a more pronounced blood pressure reduction after 1 month and 1 year $(P<0.001$, Figure 2). ${ }^{10}$ Amlodipine was superior to valsartan on the incidence of myocardial infarction, which may also have been a blood pressure-related effect. However, valsartan reduced the new onset of diabetes significantly $(13.1 \%$ vs $16.4 \%$, hazard ratio [HR]: $0.77,95 \% \mathrm{CI}: 0.69-0.86, P<0.0001)$, which is in line with the results of the LIFE trial.

Similar to VALUE, the Kyoto Heart Study included patients $(n=3031)$ with hypertension and additional risk factors. ${ }^{11}$ The trial was placebo-controlled, with valsartan used as an add-on treatment. Although baseline blood pressure was similar $(157 / 88 \mathrm{mmHg})$, in-trial blood pressure reductions were somewhat greater than those seen in the VALUE trial $(-24 /-12 \mathrm{mmHg})$, but without any difference between the treatment groups. Moreover, concomitant antihypertensive treatment was comparable in both treatment arms; thus results might be interpreted as blood pressure-independent effects of the ARB. Add-on treatment with valsartan significantly reduced the primary composite endpoint (HR: $0.55,95 \% \mathrm{CI}$ : $0.42-0.72, P=0.00001$ ) as well as rate of stroke (HR: 0.55 , 95\% CI: $0.3-0.9, P=0.01488)$, and again new onset of diabetes (HR: $0.67,95 \%$ CI: $0.5-0.9, P=0.02817$ ). However, the results are limited by the open-label design, particularly given that the superior efficacy regarding the primary composite

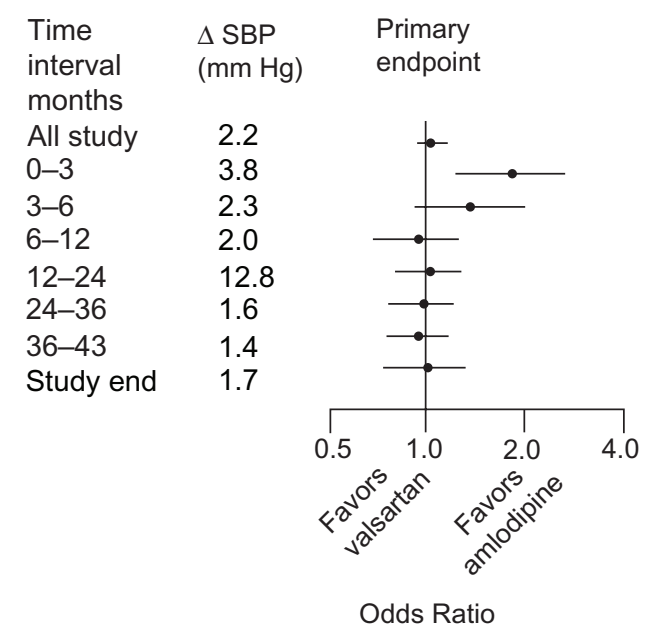

Figure 2 Differences $(\Delta)$ in SBP between treatment groups in the VALUE trial during consecutive time periods in the study, with odds ratios for primary endpoint cardiac morbidity and mortality.

Abbreviations: SBP, systolic blood pressure; VALUE, Valsartan Antihypertensive Long-term Use Evaluation. endpoint was strongly driven by the relatively soft endpoints of angina and transient ischemic attack.

\section{High cardiovascular risk}

A very substantial population is those patients who have controlled blood pressure, but remain at high cardiovascular risk due to the presence of atherosclerotic lesions or even patients with a prior cardiovascular event but without heart failure. Moreover, approximately $50 \%$ of patients with type 2 diabetes have microvascular or macrovascular complications, placing them in the high-risk category for a future cardiovascular event. ${ }^{12}$

Two ARB trials have recruited patients from this stage of the cardiovascular continuum. The Ongoing Telmisartan Alone and in Combination with Ramipril Global Endpoint Trial (ONTARGET) program recruited 25,620 patients with coronary, peripheral, or cerebrovascular disease, or diabetes with end-organ damage to receive treatment with telmisartan, ramipril, or the combination. ${ }^{13}$ It was thus notable for being the first major trial to compare an ARB with another blocker of the renin-angiotensin system (ramipril), which might be suggested as a gold standard for secondary prevention in patients with high cardiovascular risk in view of the Heart Outcomes Prevention Evaluation (HOPE) trial. ${ }^{14}$ In ONTARGET, telmisartan and ramipril were similarly effective on the primary composite endpoint of cardiovascular death, myocardial infarction, stroke, or hospitalization for heart failure (1423 of 8542 patients [16.7\%] vs 1412 of 8576 patients [16.5\%], RR: 1.01, 95\% CI 0.94-1.09; Figure 3), as well as for the individual component of cardiovascular death (7.0\% vs 7.0\%, RR: 1.00 , 95\% CI 0.89-1.12), and other outcomes of non-cardiovascular death $(4.6 \%$ vs $4.8 \%$, RR: $0.96,95 \%$ CI $0.83-1.10)$ and death from any cause $(11.6 \%$ vs $11.8 \%$, RR: $0.98,95 \%$ CI $0.90-1.07)$. The combination was no more effective and was associated with significantly increased side effects.

The Telmisartan Randomized Assessment Study in ACE-I Intolerant Subjects with Cardiovascular Disease (TRANSCEND) trial had a design similar to that of ONTARGET, but included angiotensin-converting enzyme inhibitor-intolerant patients, who are considered likely to represent $15 \%-20 \%$ of the general population. Thus, patients in TRANSCEND received add-on treatment with telmisartan compared with placebo. There was a trend to superiority on the primary composite endpoint (465 of 2954 patients [15.7\%] vs 504 of 2972 patients [17.0\%], HR: $0.91,95 \%$ CI: $0.80-1.05)$, with the individual component of cardiovascular death being similar $(7.7 \%$ vs $7.5 \%$, 


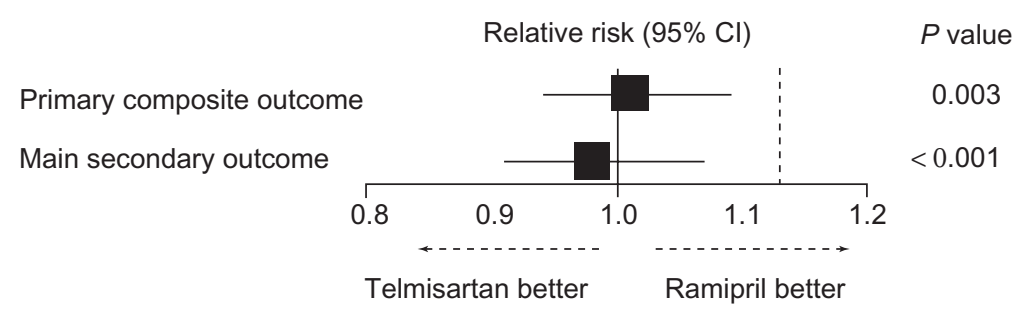

Figure 3 Relative risk of the primary outcome and of the main secondary outcome in the ONTARGET trial. The dotted line indicates the noninferiority margin. Note: The $P$ value indicates noninferiority of telmisartan compared with ramipril.

Abbreviations: $\mathrm{Cl}$, confidence interval; ONTARGET, Ongoing Telmisartan Alone and in Combination with Ramipril Global Endpoint Trial.

HR: $1.03,95 \%$ CI: $0.85-1.24, P=0.778)$. The secondary endpoint (that matches the primary endpoint in HOPE and did not include hospitalization for heart failure) reached statistical significance $(13.0 \%$ vs $14.8 \%$, HR: $0.87,95 \%$ CI: $0.76-1.00, P=0.048)$. Total mortality was similar in the telmisartan and placebo groups (12.3\% vs $11.7 \%$, HR: 1.05 , 95\% CI: $0.91-1.22, P=0.491) .{ }^{15}$ As in ONTARGET, concomitant medical treatment was excellent in TRANSCEND, with around $55 \%$ of patients receiving statins and $58 \%$ being treated with a $\beta$-blocker, respectively. Compared with the HOPE trial, background medical treatment improved markedly in ONTARGET as well as in TRANSCEND, although the time difference was only 8 years (Table 1$).{ }^{16}$

The ONTARGET and TRANSCEND study populations differed from those in the LIFE, VALUE, and Kyoto Heart trials because they had well controlled baseline blood pressure and higher prevalence of cardiovascular disease (Table 2). This is reflected in the blood pressure reductions from baseline during the trial, which were relatively small in ONTARGET, at around $-7 /-5 \mathrm{mmHg}$, compared with reductions of around $-15 /-9 \mathrm{mmHg}$ in VALUE, $-24 /-12 \mathrm{mmHg}$ in Kyoto Heart, and $-30 /-17 \mathrm{mmHg}$ in LIFE.

The Randomized Olmesartan and Diabetes Microalbuminuria Prevention (ROADMAP) trial was a recent placebo-controlled study in patients without hypertension but with diabetes and additional risk factors, thus placing

Table I Baseline medication usage (\% of patients) in the HOPE, ${ }^{14}$ ONTARGET, ${ }^{15}$ and TRANSCEND ${ }^{\circledR}$ trials $^{16}$

\begin{tabular}{llll}
\hline & HOPE & ONTARGET & TRANSCEND \\
\hline Recruitment completed & 1995 & 2003 & 2004 \\
ACE inhibitors & 11.6 & 57.5 & 58.1 \\
B-blockers & 39.5 & 56.9 & 57.6 \\
Diuretics & 15.1 & 27.9 & 32.6 \\
Oral anticoagulants & 3.8 & 7.6 & 7.2 \\
Statins & 28.9 & 60.7 & 54.4 \\
\hline
\end{tabular}

Abbreviations: ACE, angiotensin-converting enzyme; HOPE, Heart Outcomes Prevention Evaluation; ONTARGET, Ongoing Telmisartan Alone and in Combination with Ramipril Global Endpoint Trial; TRANSCEND, Telmisartan Randomized Assessment Study in ACE-I Intolerant Subjects with Cardiovascular Disease. them at increased cardiovascular risk. ${ }^{17}$ The primary endpoint was the time to first onset of microalbuminuria, which after adjusting for blood pressure differences was nonsignificant. The secondary endpoint was a composite of cardiovascular complications or death from cardiovascular causes, and there was no difference between olmesartan and placebo (which was reached by 96 of 2232 patients [4.3\%] in the olmesartan group and by 94 of 2215 patients [4.2\%] in the placebo group; HR: $1.00,95 \%$ CI: $0.75-1.33, P=0.99)$. However, deaths from cardiovascular causes were significantly higher with olmesartan $(\mathrm{n}=15,0.7 \%)$ than with placebo $(\mathrm{n}=3,0.1 \%$; HR: $4.94,95 \%$ CI: $1.43-17.06, P=0.01$ ), although deaths from any cause were not significantly different (26 [1.2\%] patients vs 15 [1.7\%] patients, HR: 1.70, 95\% CI: 0.90-3.22, $P=0.10)$. A similar excess cardiovascular mortality with olmesartan was seen in Olmesartan Reducing Incidence of End Stage Renal Disease in Diabetic Nephropathy Trial (ORIENT).${ }^{18}$ The cause of this excess mortality is unknown, but these results suggest that olmesartan should be used with caution in patients at high cardiovascular risk.

\section{Heart failure or left ventricular systolic dysfunction}

Compared with those at an early stage of the cardiovascular continuum, patients with heart failure tend to be older, and often have several comorbidities. ${ }^{19}$ Two trials have compared ARBs with captopril in patients with heart failure or left ventricular dysfunction after a recent myocardial infarction ( $\leq 10$ days). The Valsartan in Acute Myocardial Infarction Trial (VALIANT) compared valsartan, captopril, and the combination in nearly 15,000 patients, with an average follow-up of 2 years. ${ }^{20}$ The Optimal Therapy in Myocardial Infarction with the Angiotensin II Antagonist Losartan (OPTIMAAL) trial compared a relatively low dose of losar$\tan (50 \mathrm{mg}$ once daily) with captopril $50 \mathrm{mg}$ three times daily in 5477 patients and had a mean follow-up of 2.7 years. ${ }^{21}$ Whereas VALIANT demonstrated clear equivalence on the primary endpoint (all-cause mortality) between valsartan 
Table 2 Baseline characteristics of ARB trials in patients at the early-to-mid stages of the CV continuum

\begin{tabular}{lllllll}
\hline & Hypertension (\%) & Baseline SBP/DBP $(\mathbf{m m H g})$ & CAD (\%) & LVH (\%) & Stroke/TIA (\%) & PAD (\%) \\
\hline LIFE & 100 & $174 / 98$ & 16 & 100 & 8 & 6 \\
VALUE & 100 & $155 / 87$ & 46 & 6 & 20 & 14 \\
Kyoto Heart & 100 & $157 / 88$ & 23 & 8 & 4 & N/A \\
ONTARGET & 69 & $142 / 82$ & 74 & 13 & 20 & 14 \\
TRANSCEND & 76 & $141 / 82$ & 74 & 13 & 22 & 11 \\
\hline
\end{tabular}

Abbreviations: CAD, coronary artery disease; DBP, diastolic blood pressure; LVH, left ventricular hypertrophy; NA, data not available; PAD, peripheral arterial disease; SBP, systolic blood pressure; TIA, transient ischemic attack; LIFE, Losartan Intervention For Endpoint; VALUE, Valsartan Antihypertensive Long-term Use Evaluation; ONTARGET, Ongoing Telmisartan Alone and in Combination with Ramipril Global Endpoint Trial; TRANSCEND, Telmisartan Randomized Assessment Study in ACE-I Intolerant Subjects with Cardiovascular Disease.

and captopril, there was a trend for superiority of captopril over losartan in OPTIMAAL (HR: 1.13, 95\% CI: 0.99-1.28, $P=0.07)$. Different results for losartan and valsartan might be explained by a lower cardiovascular risk of patients in OPTIMAAL than that of those enrolled in VALIANT. Furthermore, differences in dosing and titration regimens may have influenced the results, because blood pressure reductions in OPTIMAAL were lower than with valsartan in VALIANT.

The Evaluation of Losartan in the Elderly II (ELITE II) trial compared losartan $50 \mathrm{mg}$ once daily with captopril $50 \mathrm{mg}$ three times daily in patients with chronic heart failure, and found both substances to be similarly effective in preventing all-cause mortality; however, there was a tolerability advantage to losartan. ${ }^{22}$ More recently, the Heart Failure Endpoint Evaluation of AII-Antagonist Losartan (HEAAL) study, which examined the effects of high-dose vs low-dose losartan on clinical outcomes in patients with heart failure, found that losartan $150 \mathrm{mg}$ /day was more effective than $50 \mathrm{mg} /$ day in reducing death or admission for heart failure (HR: 0.9, 95\% CI: 0.83-0.99, $P=0.027),{ }^{23}$ which suggests that losartan was underdosed in ELITE II. The beneficial effects of high-dose losartan were limited by a significantly increased number of side effects (renal impairment, hyperkalemia, hypotension), although discontinuation of study drug was similar for both treatment regimens.

In the Candesartan in Heart Failure: Assessment of Reduction in Mortality and Morbidity (CHARM) study, candesartan $32 \mathrm{mg} /$ day reduced both cardiovascular deaths and hospital admissions compared with standard treatment (Figure 4). However, ARBs have not been found to benefit patients with heart failure with preserved ejection fraction in the CHARM-Preserved stratum, which is in line with the results of the Irbesartan in Heart Failure with Preserved Systolic Function (I-PRESERVE) trial. ${ }^{24,25}$

In patients with heart failure on hemodialysis, telmisartan $80 \mathrm{mg} /$ day reduced all-cause mortality (HR: 0.51, 95\%
CI: $0.32-0.82, P<0.01)$ compared with best usual care, as well as hospital admissions for chronic heart failure and cardiovascular deaths. ${ }^{26}$

\section{Safety of ARBs}

A meta-analysis of five ARB trials involving 61,590 patients with new cancer data available found ARBs to be associated with a modestly increased risk of new-onset cancer $(7.2 \%$ vs 6.0\%, RR: 1.08, 95\% CI: $1.01-1.15, P=0.016) .{ }^{27}$ However, this meta-analysis was criticized on methodological grounds, particularly given that it did not include some important trials. ${ }^{28}$ More recently, the most comprehensive assessment of ARBs and cancer to date has analyzed a total of 15 trials involving 138,769 patients. ${ }^{29}$ ARBs as a class were not associated with increased risk of cancer compared with comparators (odds ratio [OR]: 1.00, 95\% CI: 0.95-1.04), and there was also no increase in cancer risk with ARBs in combination with angiotensin-converting enzyme inhibitors. No individual ARB was associated with an increased risk of cancer. On the basis of existing clinical trials, there does not seem to be any reason to suspect ARBs of increasing the risk of cancer. By contrast, the cardiovascular disease benefits of these agents are well established.

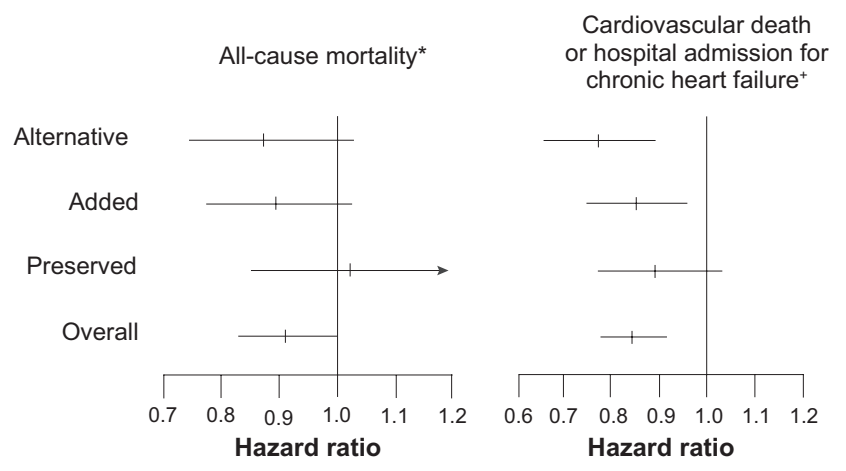

Figure 4 Hazard ratio (candesartan vs control) of the all-cause mortality and CV death or hospital admission for chronic HF in CHARM-Overall, -Preserved, -Added and -Alternative.

Notes: $P$ values for heterogeneity: $* P=0.37 ;{ }^{\dagger} P=0.33$.

Abbreviations: CV, cardiovascular; HF, heart failure; CHARM, Candesartan in Heart Failure: Assessment of Reduction in Mortality and Morbidity. 


\section{Combination of ARBs and angiotensin-converting enzyme inhibitors}

ONTARGET demonstrated no benefit from adding ARBs and angiotensin-converting enzyme inhibitors in patients at high cardiovascular risk without heart failure. For patients with chronic heart failure, results of the clinical trials diverge.

In the Valsartan Heart Failure Trial (Val-HeFT), additional treatment with valsartan $160 \mathrm{mg}$ /day in patients with chronic heart failure, who were mostly (93\%) being treated with an angiotensin-converting enzyme inhibitor, reduced hospital admission but did not reduce mortality. ${ }^{30}$ Similarly, VALIANT found no benefit on mortality, and an increase in adverse events from combination treatment with valsartan and captopril in post-myocardial infarction patients. ${ }^{20}$

The CHARM-Added stratum of the CHARM trials found a significant benefit of adding candesartan to ongoing angiotensin-converting enzyme inhibitor therapy on the composite of cardiovascular death or hospital admission for chronic heart failure ${ }^{31}$ This is suggestive of a difference between candesartan and valsartan due to dosing. Whereas CHARM-Added used the same target dose of candesartan in the monotherapy and combination arms, the combination arm in VALIANT received half the valsartan dose that the monotherapy arm received. However, patients in Val-HeFT received the higher valsartan dose of $160 \mathrm{mg}$ without an effect on mortality.

\section{Clinical implications}

There is strong evidence suggesting a reduction of cardiovascular risk when adding an ARB to evidence-based concomitant drug therapy. Candesartan and valsartan, and in part losartan at higher doses, have shown efficacy in patients with heart failure, while telmisartan has been shown to be effective in a broad range of patients at high cardiovascular risk due to existing atherosclerotic disease or diabetes with end-organ damage.

For patients in whom raised blood pressure represents a major contributory factor to their cardiovascular risk, such as those recruited into the LIFE and VALUE trials, the evidence for specific benefit with ARBs is less clear, due to the comparator substance in LIFE and more pronounced blood pressure reduction by amlodipine in the VALUE trial. Nevertheless, in these trials, ARBs were well or even better tolerated and significantly reduced the frequency of new-onset diabetes.

One clinical question remains: Should a specific ARB be selected, or can a class effect be assumed in patients at high risk of cardiovascular disease? Interpretation and extrapolation from ARB outcome trials remains difficult, because different populations with different background therapies were enrolled in those trials. Although differences in the outcomes of the various trials might hint towards possible intrinsic differences in efficacy, this remains speculative given that there are no head-to-head trials comparing different ARBs in cardiovascular risk patients.

Given the evidence of potentially important pharmacological differences, a precautionary approach should be adopted, with an ARB selected according to evidence from clinical trials, which directly inform their respective indications for clinical use, unless compelling reasons exist for selection of another drug.

\section{Conclusion}

Over the past decade, the 12 ARB outcome trials reviewed here have provided 384,000 years of patient data. These major cardiovascular outcomes studies offer unequivocal evidence that ARBs reduce cardiovascular risk. What remains uncertain is the extent to which reduction in risk differs or exceeds that available with other antihypertensive classes, due to pleiotropic effects of ARBs, and the degree to which the known pharmacological differences justify intraclass preferences.

ARBs have therefore justified their central role along with angiotensin-converting enzyme inhibitors in the management of cardiovascular risk, by demonstrating their efficacy along the cardiovascular continuum, from patients with risk factors, through those at high cardiovascular risk, to patients with heart failure. A number of factors should be considered in the selection of an antihypertensive agent to reduce cardiovascular events: antihypertensive efficacy, of course, but also factors such as cardiovascular prevention beyond lowering of blood pressure alone, tolerability, and the potential for combination therapy.

\section{Disclosure}

Both authors have received grants from Boehringer Ingelheim, Germany, that are not related to the present work.

\section{References}

1. Allender S, Scarborough P, Peto V, et al. European Cardiovascular Disease Statistics. 2008 ed. Available at: http://www.heartstats.org. Accessed June 2, 2010.

2. Werner C, Baumhakel M, Teo KK, et al. RAS blockade with ARB and ACE inhibitors: current perspective on rationale and patient selection. Clin Res Cardiol. 2008;97:418-431.

3. Bakris G, Böhm M, Dagenais G, et al. Cardiovascular protection for all individuals at high risk: evidence-based best practice. Clin Res Cardiol. 2008;97:713-725. 
4. Friedrich EB, Teo KK, Böhm M. ACE inhibition in secondary prevention: are the results controversial? Clin Res Cardiol. 2006;95: 61-67.

5. Baumhakel M, Böhm M. Telmisartan prevents cardiovascular events in a broad group of at-risk patients. Expert Opin Pharmacother. 2009; 10:3113-3117.

6. Nickenig G, Harrison DG. The AT(1)-type angiotensin receptor in oxidative stress and atherogenesis: part I. Oxidative stress and atherogenesis. Circulation. 2002;105:393-396.

7. Dzau VJ, Antman EM, Black HR, et al. The cardiovascular disease continuum validated: clinical evidence of improved patient outcomes. Part I. Pathophysiology and clinical trial evidence (risk factors through stable coronary artery disease). Circulation. 2006;114:2850-2870.

8. Dahlöf B, Devereux RB, Kjeldsen SE, et al. LIFE Study Group. Cardiovascular morbidity and mortality in the Losartan Intervention For Endpoint reduction in hypertension study (LIFE): a randomised trial against atenolol. Lancet. 2002;359:995-1003.

9. Mancia G, De Backer G, Dominiczak A, et al. 2007 guidelines for the management of arterial hypertension: the Task Force for the Management of Arterial Hypertension of the European Society of Hypertension (ESH) and of the European Society of Cardiology (ESC). J Hypertens. 2007;25:1105-1187.

10. Julius S, Kjeldsen SE, Weber M, et al. Outcomes in hypertensive patients at high cardiovascular risk treated with regimens based on valsartan or amlodipine: the VALUE randomised trial. Lancet. 2004; 363:2022-2031.

11. Sawada T, Yamada H, Dahlof B, et al. Effects of valsartan on morbidity and mortality in uncontrolled hypertensive patients with high cardiovascular risks: KYOTO Heart Study. Eur Heart J. 2009;30: 2461-2469.

12. International Diabetes Federation. The Diabetes Atlas. 3rd ed, 2006. Available at: http://www.eatlas.idf.org. Accessed June 2, 2010.

13. Yusuf S, Teo KK, Pogue J, et al. Telmisartan, ramipril, or both in patients at high risk for vascular events. N Engl J Med. 2008;358:1547-1559.

14. Yusuf S, Sleight P, Pogue J, et al. Effects of an angiotensinconverting-enzyme inhibitor, ramipril, on cardiovascular events in high-risk patients. The Heart Outcomes Prevention Evaluation Study Investigators. N Engl J Med. 2000;342:145-153.

15. Yusuf S, Teo K, Anderson C, et al. Effects of the angiotensin-receptor blocker telmisartan on cardiovascular events in high-risk patients intolerant to angiotensin-converting enzyme inhibitors: a randomised controlled trial. Lancet. 2008;372:1174-1183.

16. Teo K, Yusuf S, Anderson C, et al. Rationale, design, and baseline characteristics of 2 large, simple, randomized trials evaluating telmisartan, ramipril, and their combination in high-risk patients: the Ongoing Telmisartan Alone and in Combination with Ramipril Global Endpoint Trial/Telmisartan Randomized Assessment Study in ACE Intolerant Subjects with Cardiovascular Disease. (ONTARGET ${ }^{\circledR /}$ TRANSCEND ${ }^{\circledR}$ ) trials. Am Heart J. 2004;148:52-61.

17. Haller $\mathrm{H}$, Ito $\mathrm{S}$, Izzo JL, et al. Olmesartan for the delay or prevention of microalbuminuria in type 2 diabetes. $N$ Engl J Med. 2011;364: 907-917.
18. Food and Drug Administration. FDA drug safety communication: ongoing safety review of Benicar and cardiovascular events. June 11, 2010. Available at: http://www.fda.gov/Drugs/DrugSafety/Post marketDrugSafetyInformationforPatientsandProviders/ucm 215222. htm. Accessed April 5, 2011.

19. Owan TE, Hodge DO, Herges RM, et al. Trends in prevalence and outcome of heart failure with preserved ejection fraction. $N$ Engl $J$ Med. 2006;355:251-259.

20. Pfeffer MA, McMurray JJ, Velazquez EJ, et al. Valsartan, captopril, or both in myocardial infarction complicated by heart failure, left ventricular dysfunction, or both. N Engl J Med. 2003;349:1893-1906.

21. Dickstein K, Kjekshus J; OPTIMAAL steering committee. Effects of losartan and captopril on mortality and morbidity in high-risk patients after acute myocardial infarction: the OPTIMAAL randomised trial. Lancet. 2002;360:752-760.

22. Pitt B, Poole-Wilson P, Segal R, et al. Effect of losartan compared with captopril on mortality in patients with symptomatic heart failure: randomised trial - the Losartan Heart Failure Survival Study ELITE II. Lancet. 2000;355:1582-1587.

23. Konstam MA, Neaton JD, Dickstein K, et al. Effects of high-dose versus low-dose losartan on clinical outcomes in patients with heart failure (HEAAL study): a randomised, double-blind trial. Lancet. 2009;374:1840-1848.

24. Yusuf S, Pfeffer MA, Swedberg K, et al. Effects of candesartan in patients with chronic heart failure and preserved left-ventricular ejection fraction: the CHARM-Preserved Trial. Lancet. 2003;362:777-781.

25. Massie BM, Carson PE, McMurray JJ, et al. Irbesartan in patients with heart failure and preserved ejection fraction. N Engl J Med. 2008; 359:2456-2467.

26. Cice G, Di Benedetto A, D'Isa S, et al. Effects of telmisartan added to angiotensin-converting enzyme inhibitors on mortality and morbidity in hemodialysis patients with chronic heart failure: a double-blind, placebo-controlled trial. J Am Coll Cardiol. 2010;56:1701-1708.

27. Sipahi I, Debanne SM, Rowland DY, et al. Angiotensin-receptor blockade and risk of cancer: meta-analysis of randomized controlled trials. Lancet Oncol. 2010;11:627-636.

28. Julius S, Kjeldsen S, Weber M. Angiotensin-receptor blockade and the risk of cancer. Omission of VALUE trial data invalidates conclusions. Lancet Oncol. 2010;11:820-821.

29. The ARB Trialists Collaboration. Effects of telmisartan, irbesartan, valsartan, candesartan, and losartan on cancers in 15 trials enrolling 138769 individuals. J Hypertens. 2011;29:623-635.

30. Cohn JN, Tognoni G; Valsartan Heart Failure Trial Investigators. A randomized trial of the angiotensin-receptor blocker valsartan in chronic heart failure. N Engl J Med. 2001;345:1667-1675.

31. McMurray JJ, Ostergren J, Swedberg K, et al. Effects of candesartan in patients with chronic heart failure and reduced left-ventricular systolic function taking angiotensin-converting-enzyme inhibitors: the CHARM-Added trial. Lancet. 2003;362:767-771.
Vascular Health and Risk Management

\section{Publish your work in this journal}

Vascular Health and Risk Management is an international, peerreviewed journal of therapeutics and risk management, focusing on concise rapid reporting of clinical studies on the processes involved in the maintenance of vascular health; the monitoring, prevention and treatment of vascular disease and its sequelae; and the involvement of

\section{Dovepress}

metabolic disorders, particularly diabetes. This journal is indexed on PubMed Central and MedLine. The manuscript management system is completely online and includes a very quick and fair peer-review system, which is all easy to use. Visit http://www.dovepress.com/ testimonials.php to read real quotes from published authors. 